Ioan Klajn

Beograd

\title{
INTORNO ALLA DEFINIZIONE DEL PRONOME
}

1. E probabile che la distinzione tra le varie «parti del discorso» esisterà sempre in qualunque grammatica, quali che siano $i$ suoi presupposti metodologici. E una distinzione che scaturisce dall'analisi morfologica, ma è anche una delle ragioni d'essere di quest'analisi, il ponte che la lega alla sintassi e alla semantica. La nozione delle parti del discorso ci aiuta a scoprire la natura e il funzionamento del linguaggio, mostrando che esso non è un ammasso di elementi indifferenziati, bensì un sistema, un meccanismo in cui ogni elemento ha una forma e un compito particolari.

La divisione tradizionale in nove o dieci parti del discorso, più o meno uguale in tutte le lingue europee, è stata oggetto di numerose e non infondate critiche all'epoca dello strutturalismo. Purtroppo, delle nuove classificazioni proposte per sostituirla nessuna ha attecchito, anzi tutte sono rimaste lettera morta. Riforme di questo genere furono tentate, ognuna su basi diverse, da FRIES (si veda la bibliografia alla fine), da HOCKETT, e già prima da JESPERSEN (96 ss.). Anche a prescindere dalla validità teorica di queste classificazioni, si può constatare che sono fallite per la loro assoluta inferiorità terminologica rispetto alla classificazione tradizionale. È difficile immaginare una qualsiasi indagine linguistica o grammaticale in cui non si faccia costante uso di termini come «verbo», «preposizione», «sostantivato», «aggettivato», «deverbale», «funzione avverbiale», «forma pronominale», ecc. Malgrado i possibili difetti, questa è una terminologia ricca, flessibile e di facile comprensione. Tentare di sostituirla con espressioni come quelle di Fries, «Class 3», «Group E» e simili, sarebbe lo stesso che parlare in cifra.

Nessuna meraviglia, quindi, che i trasformazionalisti degli ultimi anni siano tornati alla classificazione abituale e che adoperino $i$ termini di sempre - «Noun», «Verb», «Adverb», «Preposition» ecc. Bisogna riconoscere che $\mathrm{i}$ grammatici antichi, alla loro maniera intuitiva ed empirica, sono pur riusciti a individuare le categorie che sono veramente fondamentali sia dal punto di vista dell'indagine scientifica sia da quello del funzionamento della lingua. Questo non toglie che la definizione 
delle parti del discorso debba essere posta su basi diverse da quelle della grammatica tradizionale, la quale peccava in questo campo di indeterminatezza e soprattutto di incoerenza. I glottologi moderni (p. es. LYONS, 317-320; ROBERTS, 10-13, 18) hanno osservato più volte che le definizioni tradizionali si basavano su criteri disparati: a volte sul significato, a volte sulla forma, a volte infine sulla funzione sintattica. Infatti, basta vedere la grammatica italiana di Battaglia e Pernicone, dove i sostantivi sono «le parole che noi adoperiamo... per indicare persone, animali, cose» (definizione semantica), mentre l'aggettivo «serve a qualificare o specificare il nome» (definizione funzionale). Il rigore scientifico richiederebbe che si trovasse un criterio unico per la definizione di tutte le parti del discorso. Ma quale potrebbe essere questo criterio?

Non il significato, perché nessuna parte del discorso ha un suo campo semantico esclusivo (bianco, bianchezza e biancheggiare indicano tutti ugualmente una «qualità»; del tutto impossibile sarebbe poi definire il significato comune a tutte le congiunzioni o a tutti gli avverbi). Non la forma, perché solo raramente le parole invariabili hanno un suffisso distintivo o quelle variabili una flessione specifica ed esclusiva (tranne i verbi, che sono comunque la categoria più facilmente individuabile). Il criterio più sicuro potrebbe essere la funzione sintattica, ma questo concetto apparentemente unitario nasconde in realtà tanti fenomeni diversi: per i verbi o i sostantivi si tratta di funzione nella frase, per gli aggettivi o gli avverbi della specificazione di un'altra parola, le congiunzioni hanno una funzione di collegamento, le preposizioni una «relazionale», ecc.

II Fries credette di aver trovato la soluzione respingendo tutti e tre questi criteri e adottando invece quello puramente formale della nistribuzione o collocazione. Egli infatti classifica le parole in base alla loro capacità di occupare determinati posti in alcune frasi-campione («test frames»). Ma il risultato - una divisione in 19 «classi» e «gruppi», dove articoli, numerali e pronomi possessivi sono ammassati nello stesso gruppo, mentre there o please hanno ciascuno una categoria tutta per sé può dirsi senz'altro un fallimento. Né è migliore la classificazione di Hockett, che ha sette classi per i soli nomi, aggettivi e verbi, ma relega tutte le altre parole indistintamente in un'ottava classe detta "particles»! Molta fortuna hanno avuto le classificazioni che potrebbero dirsi «graduali» e delle quali è prototipo quella di Jespersen (l. cit.): i sostantivi sono parole «primarie», gli aggettivi sono «secondari» in quanto specificano o modificano i sostantivi, gli avverbi modificano gli aggettivi e quindi sono «terziari». Non dissimile - sebbene basata sulla sintassi della proposizione anziché sui soli rapporti sintagmatici - è la classificazione trasformazionalista di LYONS (320-327). Tutti questi schemi hanno però un difetto comune: distinguono molto bene le categorie principali (sostantivi, aggettivi, verbi e avverbi) ma lasciano nell'ombra 
le altre, quelle più problematiche, cioè i pronomi, i numerali, le congiunzioni, le preposizioni.

Dopo tutti questi tentativi più o meno falliti ci sembra lecito arrivare alla seguente conclusione: è illusorio cercare un criterio unico per le parti del discorso, essendo queste una nozione complessa in cui si sommano e incrociano fattori molto diversi. Bisogna invece combinare $i$ quattro criteri suaccennati (significato, forma, funzione, distribuzione) per raggiungere un certo grado di coerenza. Così p. es., volendo definire i sostantivi e gli aggettivi italiani, daremo degli uni e degli altri una descrizione quadruplice:

SOSTANTIVI: Funzione: soggetto, complemento oggetto, ecc. Distribuzione: tra l'articolo e il verbo. Forma: flessione singolare-plurale, genere fisso. Significato: persone, cose, ecc.

AGGETTIVI: Funzione: qualificano il sostantivo. Distribuzione: tra l'articolo e il sostantivo. Forma: genere variabile, comparazione. Significato: qualità, ecc.

Come si vede abbiamo notato soltanto le proprietà tipiche, sufficienti a individuare la categoria: non è necessario elencare tutte le funzioni del sostantivo nella frase, tutte le sue collocazioni, oppure il fatto che anche l'aggettivo ha forme per il singolare e il plurale.

Quanto al significato, è ovvio che formulazioni come quelle accennate qui non potranno mai essere complete né precise. Perciò sarà più utile concentrarsi su una dicotomia che ha avuto grande rilievo nella linguistica moderna: la distinzione tra «parole piene» e «parole vuote» (ossia «lessicali» e «grammaticali», «autosemantiche» e «sinsemantiche», «lessemi» e «morfemi» secondo la terminologia di MARTINET, «contentives» e «functors» secondo quella di Hockett, ecc.). Anche questa è una classificazione tutt'altro che facile, ma per ora ci basti ricordare che ci sono due modi di affrontarla. Dal punto di vista semantico, le parole «piene» (principalmente i sostantivi, gli aggettivi e i verbi) hanno un significato intrinseco, oggettivo, riferibile a fenomeni del mondo extralinguistico, mentre quelle "vuote» sono semplici strumenti grammaticali che servono a formare l'enunciato; il significato delle prime, per usare i termini del Fries, è «lessicale», quello delle altre »strutturale» (o «grammaticale» come dice la STRANG). L'altro punto di vista è quello formale: le parole «piene» costituiscono «classi aperte», numerose e sempre accrescibili con la creazione di neologismi, le parole «vuote» invece formano «sistemi chiusi», con un numero limitato di parole di cui molte in opposizione reciproca (qua-là, uno-il, ecc.). Il limite fra i due gruppi non sempre coincide con la divisione in parti del discorso, dato che p. es. i verbi sono normalmente «lessicali», ma gli ausiliari sono «grammaticali», gli avverbi sono in parte «pieni» (p. es. allegramente) in parte «vuoti» (già), mentre molte parole singole stanno a metà tra il significato «lessicale» e quello «strutturale». 
2. Di tutte le parti del discorso, nessuna è così inafferrabile, così restia a qualsiasi categorizzazione come il pronome. Ciò potrebbe derivare dal fatto che i pronomi sono un insieme di sottoclassi diverse e che per la loro stessa natura, come dice il BENVENISTE (Nature 34), «ne constituent pas une classe unitaire». Ma anche se volessimo limitarci a una sola sottoclasse, troveremmo tanti casi incerti (p. es. in italiano i pronomi che stanno tra i personali e i dimostrativi: esso, costui, colui, questi, quegli) che dovremmo rinunciare a delimitarla con precisione. Dopo più di due secoli, uno è tentato di ripetere il lamento di Buffier, grammatico francese del primo Settecento (citato dalla PINCHON, 4): «Depuis le tems qu'on parle du Pronom, on n'est point parvenu à le bien conoître: comme si sa nature étoit un de ces secrets impénétrables, qu'il n'est jamais permis d'aprofondir».

D'altra parte non si può negare che il pronome è una realtà linguistica, che ha una sua fisionomia ben concreta per quanto difficilmente definibile. Non soltanto in tutte le lingue europee (e forse in tutte le lingue del mondo) esistono i pronomi, ma esistono dappertutto gli stessi sei sottotipi (personali, possessivi, dimostrativi, relativi, interrogativi e indefiniti), che si assomigliano tra le varie lingue anche in particolari minori. Cercheremo perciò di formulare una definizione del pronome che sia valida, nei limiti del possibile, per qualsiasi lingua europea antica o moderna.

Le grammatiche scolastiche (e purtroppo, anche non poche grammatiche scientifiche del tempo più recente) scansano il problema definendo il pronome come "parola che sostituisce il nome». Ma l'identificazione del pronome col fenomeno della sostituzione linguistica, per quanto antica e radicata (e riflessa nello stesso termine pro-nomen), non è giustificata. Senza addurre $i$ vari argomenti in questo senso che abbiamo esposto in un lavoro più particolareggiato, ${ }^{1}$ accenneremo soltanto alle due ragioni principali:

a) La sostituzione non è una caratteristica esclusiva dei pronomi, poiché si ritrova in molte altre parole: un avverbio come allora può sostituire un complemento di tempo, uno come là o ci sostituisce un complemento di luogo (come negli esempi 19 e 20, più sotto), sì fa le veci di una proposizione affermativa e no di una negativa, in inglese il verbo do sostituisce altri verbi, ecc.

b) Viceversa, molti pronomi non sono sostituenti: in frasi come

(1) Io lavoro

(2) Nessuno risponde

(3) Non $t i$ amo più

(4) Con chi volevi parlare?

$1 O$ zamenicama $i$ pojmu zamenjibanja, »Anali Filološkog fakulteta«, vol. XII, Belgrado 1976. 
il pronome è l'unica parola che si può usare senza cambiare il contesto o il senso, perciò è assolutamente falso dire che esso sostituisca un nome.

3. Considerazioni simili portarono a suo tempo il BRUNOT a scartare completamente sia i termini substitut e substitution, sia la categoria del pronome, creando al suo posto due categorie nuove. I pronomi sostituenti (p. es. quelli anaforici di $3 a$ persona o i relativi) sono da lui chiamati représentants, mentre i non sostituenti (come i personali di $1 \mathrm{a}$ e $2 \mathrm{a}$ singolare e gli indefiniti) appartengono allla classe dei nominaux. Purtroppo di questi nominaux egli non dà altra definizione che «ils se rapprochent des noms sans se confondre avec eux». I termini représentant, représenter, représentation hanno avuto una certa eco nella linguistica frañcese, ma non ci sono ragioni convincenti per preferirli ai più abituali sostituente, sostituire e sostituzione. Indubbiamente lo studioso francese ha imboccato la strada giusta, ma non è riuscito a risolvere il problema del pronome.

A nostro avviso sarebbe più fruttuoso raffigurare $t u t t i$ i pronomi, sostituenti e no, come una specie di «nominaux» (anche senza adottare un termine di questo tipo: il vecchio termine pronome può benissimo essere mantenuto, purché non venga interpretato etimologicamente come «sostituente del nome»). Si potrebbe proporre, cioè, una definizione di questo genere: Il pronome è una parolia che ha la stessa funzione sintattica del sostantioo, ma che non è un sostantioo. Se il pronome è un sostituente, avrà in comune col sostantivo non solo la funzione sintattica ma anche il contesto. Per i pronomi non sostituenti, come quelli delle frasi $(1-4)$, il contesto è diverso, ma la funzione sintattica è quella propria dei sostantivi: soggetto in (1) e (2), complemento oggetto in (3), complemento preposizionale in (4), ecc.

Resta da precisare la seconda parte della definizione: come stabilire che la parola in questione «non è un sostantivo»? L'indizio più sicuro è quello distribuzionale: $i$ sostantivi possono essere preceduti da un determinante (articolo, dimostrativo, numerale), i pronomi no. Il criterio formale è meno attendibile, anche se nelle lingue con flessione molto ricca, p. es. il latino o le lingue slave, la declinazione dei pronomi è sempre alquanto diversa da quella dei sostantivi. Nelle lingue europee occidentali il pronome mantiene tracce della declinazione, mentre il sostantivo l'ha perduta; purtroppo questo fatto, importante per la grammatica storica, vale quasi esclusivamente per i pronomi personali. S'intende che il criterio più essenziale sarebbe quello del significato: i sostantivi sono parole «piene», i pronomi (sia pure in un senso speciale, su cui torneremo nella sezione 6) parole «vuote». Ma anziché sul contenuto semantico, necessariamente sfumato e incerto, si può fondare questa distinzione sul fatto che i pronomi, a differenza dei sostantivi, formano "sistemi chiusi», con un numero ristretto di elementi e con ovvie corrispondenze (noi-boi- 
-loro, ingl. somebody-anybody-enerybody-nobody, something-anything-everything-nothing, ecc.).

4. Ora però dobbiamo chiederci se abbiamo preso in considerazione tutte le parole che rientrano nel concetto del pronome. Non sono forse pronomi le parole sottolineate in

(5) Voglio questo cappello

(6) Quale capello vuoi?

(7) Non c'è nessun segno

(8) Ti presento mia moglie eccetera?

La grammatica scolastica italiana classificava queste parole tra gli «aggettivi» (dimostrativi, interrogativi, indefiniti, ecc.), ma è un errore che già il GOIDÃNICH (p. 21), mezzo secolo fa, cercava invano di rettificare. (Goidànich parla in questo caso di «pronomi aggiuntivi», chiamando «pronomi assoluti» quelli in funzione sostantivale.)

Le parole sottolineate in (5-8) hanno infatti la funzione sintattica degli aggettivi, cioè qualificano sostantivi. Ma se questo fatto bastasse a identificarli con gli aggettivi, allora secondo la stessa logica dovremmo dire che le parole sottolineate in (1-4) sono sostantivi, e i pronomi sparirebbero del tutto dalla lingua! In realtà $i$ due casi sono analoghi: come io, egli, nessuno ecc. hanno la funzione sintattica dei sostantivi ma non sono sostantivi, così quale, io, questo ecc. hanno funzione aggettivale ma non sono aggettivi. Essi differiscono dagli aggettivi sotto gli altri tre aspetti, cioè nella forma (non hanno comparazione, non prendono suffissi alterativi; anche la flessione è spesso diversa da quella aggettivale, priva di suffisso come nel ted. mein Garten, mein Haus rispetto a klein-er Garten, klein-es Haus, oppure con suffissi speciali come nel serbocroato oD-aj, $o n-a j$, russo et-ot), nella distribuzione (non appaiono tra l'articolo e il sostantivo, non vengono modificati dagli avrerbi, raramente vengono usati in posizione predicativa) ${ }^{2}$ e nel significato («strutturale» mentre quello degli aggettivi è «lessicale»). Ma è soprattutto l'esistenza di «sistemi chiusi» come mio-tuo-suo-nostro-bostro-loro, questo-codesto-quello, ecc., che separa i pronomi dagli aggettivi, «classe aperta».

La categoria dei pronomi, dunque, comprende oltre a quelli «sostantivali» (o assoluti) anche quelli «aggettivali» (o aggiuntivi). Perciò allargheremo la definizione data nel paragrafo precedente e diremo:

2 Queste differenze non sono assolute: valgono quasi totalmente per $\mathrm{i}$ dimostrativi e $i$ personali, ma non sempre per $i$ possessivi, che compaiono regolarmente in posizione predicativa, che hanno qualche raro caso di comparazione (ora sei più mio), di modificazione avverbiale (veramente nostro), e che almeno in italiano possono stare tra l'articolo e il nome. Ma per dimostrare che nonostante tutto questo i possessivi sono pronomi e non aggettivi, basta ricordare che essi si differenziano secondo le persone grammaticali - una distinzione inimmaginabile al di fuori delle due classi morfologiche dei verbi e dei pronomi. 
Il pronome è una parola che ha la stessa funzione sintattica del sostantioo o dell'aggettioo, ma che differisce da questi nella forma, nel significato e/o nella distribuzione.

5. Si obietterà forse che la definizione così formulata sarebbe applicabile anche a varie sostantivazioni (tipo $i$ se e $i$ ma, il dunque, ecc.) e aggettivazioni (tipo guerra lampo, gente bene). Ma è una difficoltà puramente formale. Una volta stabilito che se è una congiunzione, che dunque è un avverbio, ecc., diventerà chiaro che per loro la funzione sostantivale è eccezionale e provvisoria, mentre per un pronome è regolare e normale.

Ugualmente si potrebbe porre la questione dei numerali (ordinali, in alcune lingue anche cardinali), che hanno pur essi la funzione dell'aggettivo senza essere aggettivi veri. Questo è un problema molto interessante, che non possiamo tentare di risolvere in questa sede. ${ }^{3} \mathrm{Ci}$ basti ricordare che molti grammatici moderni, come Fries o Quirk, annoverano i numerali, insieme con gli articoli ed alcuni pronomi, fra i determinanti (determiners), o addirittura fra $i$ «determiner-pronouns» come la STRANG. Dal punto di vista pratico non vi è pericolo di confusione, essendo tutti i numerali immediatamente riconoscibili.

Una vera difficoltà la presentano invece $i$ «quantificatori» e gli «identificatori» come tutto, entrambi, molto, poco, tanto, quanto, troppo, altro, stesso, medesimo, tale ecc. Queste parole stanno da sempre, in tutte le lingue $e$ in tutte le grammatiche, al limite fra $i$ pronomi da una parte e gli aggettivi, i sostantivi o gli avverbi dall'altra. Nessuno dei criteri che abbiamo enumerati aiuta a classificarli con maggior precisione. Si tratta probabilmente di una indeterminatezza insita nella stessa natura di queste parole, che rappresentano veramente un anello di congiunzione tra i pronomi e le parole «piene».

6. La definizione che abbiamo formulato alla fine del paragrafo 4 è una definizione empirica: essa ci dice quello che $i$ pronomi «fanno», per aiutarci a riconoscerli e a distinguerli da altre parole simili, e come tale potrebbe essere adoperata $\mathrm{p}$. es. nell'insegnamento. Ma un linguista «puro» potrebbe sentire il bisogno di una definizione più intrinseca, una che ci dica quello che i pronomi «sono», spiegandoci la loro natura, anche

${ }^{3} \mathrm{Si}$ tratta innanzitutto di decidere se $\mathrm{i}$ numerali sono parole «piene»o «vuote», e quindi di stabilire se la loro è una «classe aperta»o un «sistema chiuso». A prima vista si direbbe che è un sistema chiuso per eccellenza, in cui è esattamente definita l'appartenenza, l'ordine e i rapporti fra gli elementi e in cui non è certo possibile la creazione di neologismi. Ma d'altra parte, lungi dall'essere limitata e poco numerosa come le altre «parole vuote», la classe è infinita, e il suo significato, se non proprio «lessicale», è certamente oggettivo e non subordinato alle necessità della grammatica. 
a costo di non essere di grande utilità pratica. Se tale è il nostro scopo, dovremo partire da presupposti affatto diversi, cercando nel significato o nel funzionamento dei pronomi un tratto comune a tutt'e sei le loro sottospecie, dai personali agli indefiniti, e assente invece da altre categorie di parole (o almeno non frequente e tipico in esse).

Sfogliando la letteratura sui pronomi troviamo indicata più volte una proprietà che sembrerebbe rispondere a questi requisiti. Il pronome, cioè, per se stesso non ha che un significato «strutturale», ma nell'uso concreto si riempie di un significato «lessicale», e ciò vale tanto per $\mathrm{i}$ sostituenti quanto per $\mathrm{i}$ non sostituenti. Il Brunot aveva già intuito una parte di questo fenomeno quando diceva che un représentant «n'éveille aucune des idées qu'éveille le nom: il est vide d'impressions, il est abstrait» (173) e similmente dei nominaux: «ils sont abstraits, n’éveillent point d'image» (63). Molto più preciso e sottile nella sua analisi, il Benveniste descrive i pronomi come «formes qui renvoient toujours et seulement à des 'individus', qu'il s'agisse de personnes, de moments, de lieux, par opposition aux termes nominaux qui renvoient toujours et seulement à des concepts ... Ces 'individus linguistiques' ... sont engendrés à nouveau chaque fois qu'une énonciation est proférée, et chaque fois ils désignent à neuf» (Problèmes 83; similmente Nature, 34-35). Il Roberts osserva: «Pronouns... do not name, as nouns do... They always derive their meaning from the context» (54). IL LO CASCIO chiama «stato semantico stabile» i connotati strutturali fissi del pronome (genere, numero, persona ecc.), mentre «l'aspetto semantico di cui ogni volta nel contesto i vari pronomi si 'riempiono'» costituisce lo «strato semantico mobile».

Come vedremo più tardi, il Beneveniste espone in questo caso una concezione più limitata di quella degli altri autori citati, più prudente e perciò più vicina al vero. Ad ogni modo, tutti questi studiosi condividono un'idea di base che potrebbe essere espressa così: i pronomi sono parole «vuote» nella langue, ma diventano «piene» nella parole. 亡̀ un fatto abbastanza distintivo e singolare, ma può davvero caratterizzare il pronome?

Gli autori sopraccitati, pur parlando generalmente del «pronome», si riferiscono in realtà ai soli pronomi personali. Bisogna vedere se lo stesso «riempimento lessicale» si verifica anche nei pronomi delle altre cinque sottoclassi.

Possiamo rispondere affermativamente per quanto riguarda i relativi, i possessivi e i dimostrativi anaforici. Analogamente a

(9) Chiamo Monica e lei (= Monica) mi risponde

si ha lo steso «riempiamento» in

(10) Chiamo Monica, la quale (= Monica) mi risponde

(11) Chiamo Monica e la sua voce (= di Monica) mi risponde

(12) Chiamo Monica e questa (= Monica) mi risponde

Le cose diventano più difficili con gli interrogativi e gli indefiniti. In 


\section{Ioan Klajn}

(13) Chi mi risponde?

(14) Ognuno mi risponde

(15) Qualcuno mi risponde

(16) Nessuno mi risponde

i pronomi rimangono vuoti, non trasmettono l'idea di qualsiasi persona o oggetto definito. Tuttavia essi non sono neutrali rispetto al concetto di riempimento lessicale. Si può tentare di metterli in relazione con esso almeno in un modo negativo o indiretto. Chi si può interpretare come una richiesta di riempimento, che sarà soddisfatta nella risposta «Monica». Ognuno indicherebbe un riempimento generalizzato (sia Monica, sia Paolo, sia Gianna...), qualcuno l'impossibilità o la sospensione del riempimento (forse Monica, forse Paolo...), nessuno il riempimento zero (non Monica, non Paolo...), ecc. Non è una interpretazione troppo convincente, ma serve a dimostrare l'esistenza di un legame tra questi pronomi (interrogativi e indefiniti) e i personali.

Purtroppo c'è una categoria di pronomi per cui la nozione del riempimento cessa affatto di funzionare, ed è quella dei dimostrativi non sostituenti e non anaforici. In una frase come

(17) Chi ha messo questo nella mia camera?

il dimostrativo questo ha un rapporto unicamente con l'oggetto in questione, non con la parola «piena« che normalmente lo designerebbe. Può essere usato anche se tale parola non esiste, cioè se si tratta di un oggetto mai visto prima o indescrivibile. Non possiede un contenuto lessicale, non lo richiede, e nemmeno lo nega: rimane assolutamente neutrale. Chi si trova accanto al parlante potrà scoprire (seguendo il suo sguardo o i suoi gesti, dunque con mezzi extralinguistici) a quale oggetto si riferisca il suo «questo», ma non verrà necessariamente a sapere un sostantivo corrispondente, e questo rimarrà per sempre «parola vuota». Lo stesso per i dimostrativi aggettivali. In

(18) Guarda com'è buffo quel bambino

quel ha un $\mathrm{r}$ if e rimento determinato dalla situazione, ma non un significato se non quello dimostrativo che possiede già da sé, indipendentemente dal contesto. Il bambino può essere biondo o piccolo o arrabbiato, ma non per questo il pronome viene a significare «biondo», «piccolo» 0 «arrabbiato».

Queste considerazioni ci portano alla conclusione che il riempimento lessicale, per quanto caratteristico, non è una proprietà immancabile dei pronomi. $D^{\prime}$ altra parte bisogna notare (senza che ciò sia necessariamento uno svantaggio) che esso non è neanche una proprietà esclusiva dei pronomi. Il Martinet (145) confronta i pronomi personali, riferentisi «selon les cas, à des personnes réelles différentes», con la parola aujourd'hui, la quale «ne se rapporte pas à la même réalité si on l'emploie le 10 décembre 1958 ou le 5 mai 1959». Forse i due casi non sono uguali, dato che oggi ha un sia pur tenue significato «lessicale». Ma se prendiamo un puro avverbio di tempo come allora, o di luogo come là, con- 
stateremo che essi, come i pronomi, sono parole «vuote» che prendono il significato dal contesto, p. es.

(19) Potrei venire in agosto, ma allora è chiuso

(20) Va' a Parigi, là troverai tutte le risposte Ad ogni modo tutte queste parole appartengono a un gruppo più largo, cioè a quelli che Jespersen chiamava «shifters», espressioni il cui significato varia con la situazione ( $p a d r e . n e m i c o$, a casa).

7. Torniamo però ai dimostrativi esemplificati in (17) e (18). Abbiamo visto che in essi manca il « riempimento lessicale» in quanto non esprimono un concetto, non lo «significano», ma si riferiscono direttamente all'oggetto in questione. Questo riferimento diretto, questa capacità di indicare da cui appunto prendono il nome di dimostrativi, potrebbe chiamarsi d e is s i. Da notare che non usiamo questo grecismo in senso la to come il Lyons (275), per il quale deixis indica qualsiasi orientamento rispetto alla situazione spaziale e temporale del discorso, ma nel senso abituale dell'aggettivo deittico (dal gr. deíknymi, mostrare), che corrisponde piuttosto al termine di Benveniste ostension («indices de l'ostension» sono «termes qui impliquent un geste désignant l'object en même temps qu'est prononcée l'instance du terme»: Problèmes, 82).

Ora, è chiaro che in nessuna lingua i dimostrativi si limitano ad accennare agli oggetti materialmente presenti (come in (17) e (18)), ma indicano anche oggetti lontani o immaginari, avvenimenti passati o futuri, idee astratte e generiche, insomma qualsiasi c on cet to presente nella mente del parlante (p. es. «Quel ramo del lago di Como», «Questo di tanta speme oggi mi resta!», «Les hommes ne pensent qu'à ça», "And that is how I became an artist»). Questa deissi concettuale non diffe-risce nella sua sostanza dall'altra, che potremmo chiamare deissi situazionale. Tra le due non c'è nessuna frattura: se il bambino della frase (18) si allontana dalla stanza, io continuerò a chiamarlo quel bambino, e userò la stessa forma se lo rievoco dopo molti anni ( Ricordi com'era buffo quel bambino?»), oppure se il bambino non è mai esistito, ma è un personaggio di un mio romanzo.

D'altra parte, niente impedisce che lo stesso concetto indicato dal pronome venga anche espresso con sostantivi o con altre parole «piene» nell'ambito dello stesso enunciato. Così in (12), dove questa si riferisce a Monica, oppure in

(21) Quale ramo del lago di Como? Quello meridionale.

(22) To be or not to be, that is the question

dove i pronomi sottolineati si riferiscono all'intero sintagma precedente. Anche in questo caso il passaggio è impercettibile, senza alcun limite reciso: allo stesso modo in cui nel primo caso il dimostrativo indicava una realtà oggettiva, nel secondo il concetto derivato da questa realtà, così adesso indica il concetto insieme con (o attraverso) le parole che lo 
esprimono. ${ }^{4}$ Questo uso anaforico del pronome, come di solito si chiama, costituisce evidentemente un terzo tipo di deissi, strettamente legato agli altri due, che potremmo denominare deissi contestuale.

Per semplificare un po' la classificazione e la terminologia, potremmo anche chiamare deissi esterna i primi due tipi (situazionale e concettuale), deissi interna il terzo (contestuale). (Per uno psicologo anche la deissi concettuale sarebbe «interna», dato che si svolge nella mente del parlante; ma dal punto di vista linguistico sono «esterni» tutti quei fatti, materiali o mentali, che non fanno parte dell'enunciato).

8. Questa duplice deissi (riferimento al mondo reale o al pensiero da una parte, agli elementi linguistici dall'altra), che abbiamo constatato tra i dimostrativi, abbraccia anche tutti gli usi possibili dei pronomi delle altre cinque sottoclassi. Essa quindi potrebbe rappresentare quel denominatore comune che stavamo cercando. Alcuni pronomi, come i relativi, servono unicamente alla deissi interna; altri oscillano tra i due tipi. Così tra $\mathrm{i}$ personali, $\mathrm{i}$ non sostituenti come io e tu funzionano da deittici esterni (si pensi allo scolaro che alza la mano esclamando «Io!», oppure al sergente che punta il dito verso uno dei soldati dicendo $\ll$ Tu!»), mentre un pronome di terza persona di solito è sostituente, quindi deittico interno (come in (9)), ma può anche essere deittico esterno, quasi uguale al dimostrativo (p. es. «t lui che chiedeva di me?» = «Eे questo qui che chiedeva di me?»). Per gli indefiniti la deissi si definirebbe in modo simile a quello che abbiamo adottato per il riempimento lessicale: qualcuno è una rinuncia a indicare, ognuno un'indicazione massimale, nessuno un'indicazione zero, ecc. Gli interrogativi come chi e quale sono anch'essi deittici in quanto «puntano» verso la risposta.

Si confermerebbe così un'altra profonda intuizione di Pier Gabriele Goidànich, il più «linguista» fra i grammatici italiani, che scriveva più di mezzo secolo fa: «I Pronomi sono le parole che servono ad indicare od accennare in varia guisa un'estistenza» (p. 21). Un corollario sarebbe che il posto della sottoclasse più importante, quella dei pronomi «per eccellenza», spetterebbe non più ai personali ma ai dimostrativi; i personali sarebbero soltanto un caso speciale di dimostrativi, orientati non più secondo le opposizioni di vicinanza-lontananza ma secondo $i$ ruoli nel processo comunicativo.

Questa interpretazione dei pronomi come parole deittiche non contrasta affatto con la concezione benvenistiana degli «individus linguisti-

4 Non importa qui stabilire quale dei due - parola o concetto - abbia la precedenza, ossia se un pronome sostituente sostituisce le parole o il loro contenuto. Si veda a questo proposito BRUNOT, 173 (secondo cui viene sostituita, anzi rappresentata, «l'idée d'ensemble»), PINCHON, 36-37 e passim (che distingue tra «représentation complète» e «représentation conceptuelle») e il nostro lavoro citato nella nota 1. 
ques», citata nel paragrafo 6 . Si noterà che il Benveniste, a differenza di Roberts o Lo Čascio, non adopera i termini «significato» o «semantico», ma unicamente $\mathrm{i}$ verbi «renvoyer» e «dèsigner». I pronomi, quindi, non hanno un significato, né intrinseco come la parole "piene», né mutuato da queste per riempimento lessicale, ma servono solo a designare, a mostrare enti individuali. Inoltre il concetto di deissi concorda perfettamente con $i$ termini e le definizioni che HJELMSLEV ha preso dalla linguistica psicologica di van Ginneken: mentre le parole piene sono caratterizzate da una «adhésion significative», il pronome è «un mot à adhésion indicative, un mot pour ainsi dire sans 'signification' proprement dite» (p. 201).

Avvertiamo infine che nemmeno la deissi serve a separare $i$ pronomi dagli avverbi del tipo qui, là, allora, essendo anche questi «parole che servono ad indicare o accennare». Per separarli è necessario tirare in ballo la funzione sintattica, che per se stessa (come abbiamo visto nel caso dei presunti «aggettivi», $\$ 4$ ) non rappresenta un criterio sufficiente, ma che in questo caso è accompagnata dalle differenze di distribuzione (Firmi con questa penna - Firmi qui con la penna) e di forma (pronomi variabili, avverbi invariabili). D'altro canto, la presenza della deissi in ambedue le categorie non può essere ignorata; non è logico chiamare «dimostrativi» questo e quello e negare la stessa denominazione a qua e là. La grammatica dovrebbe render conto delle profonde corrispondenze che si riscontrano tra pronomi e avverbi in molte lingue, p. es. in latino (hic pronome - hic, hac, huc, hinc, avverbi; iste - istic, istac, istuc, istinc; ille - illic, illac, illuc, illinc, ecc.) o serbocroato (che ha tutta una serie di coppie avverbio-pronome: kako-kakao, ikako - ika$k a b, t a k o$ - takan, onako - obakan, onako - onakab, ecc.). Ma questo dilemma, in fondo, non è che una conferma della ben nota verità: la lingua è un continuo, ogni classificazione è artificiale, e perciò una categoria va stabilita avendo in mente determinati criteri, ma non può pretendere di descrivere e riflettere ugualmente tutte le qualità degli elementi in essa contenuti.

\section{BIBLIOGRAFIA}

Benveniste, E., La nature des pronoms, in: For Roman Jakobson, a cura di M. Halle, H. G. Lunt, H. McLean, C. $\mathbb{H}$. van Schoonevold, The Hague 1956. Benveniste, E., Problèmes de linguistique générale (II), Paris 1974.

Brunot, F., La pensée et la langue, III éd. revue, Paris 1953.

Fries, C. C., The Structure of English, London 1969 (ristampa).

Goidànich, P. G., Grammatica italiana, II ed., Bologna 1919.

Hjelmslev, L., La nature du pronom, in: L. Hjelmslev, Essais linguistiques, Paris 1971.

Hockett, Ch. F., A Course in Modern Linguistics, New York 1958.

Jespersen, O., The Philosophy of Grammar, London 1955 (ristampa).

Lo Cascio, V., Strutture pronominali e verbali italiane, Bologna 1970.

Lyons, J., Introduction to Theoretical Linguistics, Cambridge 1968. 
Martinet, A., Eléments de linguistique générale, II. ed, Paris 1961.

Pinchon, J., Les pronoms adperbiaux en et $\mathrm{y}$ - Problèmes généreaux de la représentation nominale, Genève 1971.

Quirk, R., Greenbaum, S., A University Grammar of English, London 1973.

Roberts, P., Understanding Grammar, New York 1954.

Strang, B. M. H., Modern English Structure, London 1970, II ed.

\section{Rezime}

\section{O DEFINICIJI ZAMENICA}

Cetiri osnovna kriterija za definiciju vrsta reči jesu sintaktička funkcija, oblik, distribucija i značenje. Ovaj poslednji je najneodređeniji (poznata je neadekvatnost školskih definicija kao simenice označavaju osobe i stvarik, »glagoli označavaju radnje« $i$ sl.), ali postaje pouzdaniji ako ga zasnujemo na razlici između »pnnih (leksičkih) $i$ spraznih « (gramatičkih) reči, ili leksema i morfema u Martinetovom smislu.

Zamenice u ovom pogledu zadaju još veće teškoće nego ostale vrste reči. Klasična definicija po kojoj su to »reči koje zamenjuju imenice je u stvari samo na manji deo njih. U rečenici $\gg \mathrm{Ja}$ radim $\ll$, ja ne zamenjuje imenicu (jer se imenica ne bi ni mogla upotrebiti u tom kontekstu), nego samo ima sintaktičku funkciju imenice (subjekat). Slično tome, pridevske zamenice kao moj, taj, neki imaju sintaktičku funkciju prideva (određuju imenicu). Otuda se predlaže sledeća definicija: zamenice su reči koje imaju sintaktičku funkciju imenice ili prideoa, ali se od opih razlikuju po obliku, značenju i/ili distribuciji.

Nasuprot ovoj empirijskoj definiciji mogao bi se postaviti zahter za 》čistom « definicijom, koja bi otkrila neku inherentnu osobinu zamenica. Pada u oči da su zamenice same po sebi »prazne« reči, ali da se u upotrebi mnoge od njih »ispunjavaju « konkretnim leksičkim značenjem (npr. »on« = Marko, »njegov $\ll=$ Markon, »obratih se Marku, a onaj reče $\approx=$ a Marko reče, itd.). Na žalost, za druge zamenice (upitne, neodređene, pokazne neanaforične) ta osobina ne važi.

Zajedničko obeležje svih zamenica pre bi se moglo tražiti u deiksi, tj. u sposobnosti pokazivanja ili ukazivanja. Pri tom bismo pod tim terminom morali podrazumevati dve srodne pojave: spoljna deiksa je ukazivanje na predmete i bića iz materijalnog sveta $(\gg j a \ll$, $\gg$ ti $\ll$, $\gg$ moj $\ll$, dodajte mi to $)$ ili na pojmove ( Ono su bila lepa viemena«, »O tome neću da razgovaram»). Unutrašnja deiksa je ukazivanje na druge reči iz konteksta, pre svega u slučaju anaforičnih zamenica (»Obratih se $M a r k u$, a ovaj reče, »a on reče«, $k$ koji reče itd.). Upitne zamenice mogu se tumačiti kao deiksa usmerena ka odgovoru, a neodredene, zavisno od značenja, kao nemogućnost deikse (neko, nešto), nulta deiksa (niko, ništa), generalizovana deiksa (soako, soe) itd. 